\title{
Statistical Dynamics of Religions and Adherents
}

\author{
M. Ausloos and F. Petroni \\ SUPRATECS, B5, Sart Tilman, \\ B-4000 Liège, Belgium
}

October 20, 2018

\begin{abstract}
Religiosity is one of the most important sociological aspects of populations. All religions may evolve in their beliefs and adapt to the society developments. A religion is a social variable, like a language or wealth, to be studied like any other organizational parameter.

Several questions can be raised, as considered in this study: e.g. (i) from a "macroscopic" point of view : How many religions exist at a given time? (ii) from a "microscopic" view point: How many adherents belong to one religion? Does the number of adherents increase or not, and how? No need to say that if quantitative answers and mathematical laws are found, agent based models can be imagined to describe such nonequilibrium processes.

It is found that empirical laws can be deduced and related to preferential attachment processes, like on evolving network; we propose two different algorithmic models reproducing as well the data. Moreover, a population growth-death equation is shown to be a plausible modeling of evolution dynamics in a continuous time framework. Differences with language dynamic competition is emphasized.
\end{abstract}

\section{Introduction}

All features of societies (beliefs, attitudes, behaviors, languages, wealth, etc.) are due to competition [1]. Recently the dynamics of world's languages, especially on their disappearing due to competition with other languages [2] has been of interest. It is fair to examine whether such considerations can be applied to religions.

We do not enter into any discussion on the definition of a religion; we recognize that there are various denominations which can impair data gathering and subsequent analysis; like many, we admit to put on the same footing religions, philosophies, sects and rituals. Idem for adherents or adepts; there are also agnostics, atheists or "not concerned". In fact, a similar set of considerations exists when discussing languages and dialects, slangs, etc. Indeed it is expected that there are many similarities, although many are differences $\sqrt{1}$,

${ }^{1}$ If it is possible to be bilingual, it is not common to be "bireligious" 
between the diffusion, relaxation and distribution of languages and religions. What is their geographical distribution? What is their life time? How do they evolve, - from monotheism to polytheism and "backwards"? How long does an adept/adherent remain in one religion? Moreover, even though many societies are thought to form a hierarchy, due to a competition between individual concerns, as explained by Bonabeau et al. [3] or discussed by Sousa and Stauffer [4], such considerations for religion should be left for further investigation. These questions need much more reliable data than it seems available and practical at this time. Thus, let us claim that we are not interested here in religion's origin, activity, history or hierarchy, but rather in statistical physics aspects of a non-equilibrium agent based system. We will then consider as parameters the numbers of adherents of each religion, and only these numbers will be treated as physics object (and not the religions themselves).

To address these issues, we have followed classical scientific steps as in physics investigations. We have analyzed "empirical" data on the number of adherents of religions taken from two different freely available data sets.

The next scientific step is to analyze the data along statistical physics modern lines. Zipf and Pareto-like plots will be given. After deducing empirical laws, a theoretical modeling is in order. In view of the observed features, and following standard intuition, one thinks at once about two algorithmic "agent based" models, describing preferential attachment on a network, as in the Potts model [5] of magnetism or Axelrod model [1] in sociology, already applied in opinion formation studies 6 .

Thereafter studying the time evolution of several "main" religions, we observe that a microscopic interpretation is plausible along the lines of a growth Avrami equation in a continuous time framework. This equation seems more plausible than the generalized Verhulst equation for modeling the dynamics of language deaths [2] because the former allows better handling of "internal and/or external fields" (as those mentioned above, - most of them missing in language dynamics) as well as (microscopic) homogeneous and/or heterogeneous fluctuations at an early stage of evolution, - while the Verhulst equation of Abrams and Strogatz [2] is grossly empirical. Notice that languages were simulated also with other than Lotka-Verhulst-Volterra mechanisms [2]; see e.g. ref. [7.

\section{Data}

The first data set is taken from The International Data Base (IDB) [8]. Data on Religions are included in table 58 and contains information on the population of 103 nations worldwide. The surveys were carried between 1960 and 1992. In the dataset are recorded the number of adherents of 150 religions, taking into account about 2 billion people ( $1 / 3$ of the present world population).

The second data set was taken from the World Christian Encyclopedia (WCE) 9, it gives information on the number of adherents of the world's main religions and their main denominations (56 religions overall) consider- 
ing the whole world population. From this data set we have also information on changes during one century of the number of adherents of each religion from 1900 till 2000, measured over a 5 year span, with a forecast for 2025 and 2050. No need to say that further work should go back to history: the number of "religions" is highly time dependent, the more so when one distinguishes them to the level of denominations and sects; the number of adherents of a given religion is not fixed either. History is full of examples of individuals or entire groups of people changing their religion, - for various reasons: following the "leader" (e.g. Constantinus, ...) or "external pressure" (e.g. inquisition, ...) or "internal pressure" or so called adaptation under proselytism action...

One should also be aware that such surveys are biased, and are hardly snapshots of a situation like in a laboratory experiment. Yet, beside these caveats, the main difference between the two data sets is in the information they give on religions with a small number of adherents. While this information is present (even if not for all considered nations, and only partially) in the first data set, the second data set does not consider small religious groups. It is also unclear how much distinction was made in the IDB and WCE surveys concerning denominations and sects so called adstrated to the main religions.

\section{Zipf's and Pareto's distributions}

The Zipf's and Pareto's distributions are shown in figure 1 for both data sets. Recall that the Zipf distribution results from a hierarchical ranking (of the considered religions according to their number of adherents). The Pareto distribution shows instead the number of religions with a number of adherents $n$ greater than $N$ as a function of $N$. In figure 1(a) and 1(b), the Zipf and Pareto distributions are shown respectively for the first dataset while 1(c) and 1(d) show results for the second data set in different (so-called) years. It can be noticed that the Zipf distribution for both data sets can be fitted by a straight line, with different slopes, - except for the tails, i.e. where religions with a very small or high number of adherents are to be found (see caveats above). However it is remarkable that a different behavior between both data sets is found in the case of the Pareto distribution: while for the IDB data set, Fig 1(b), it can be seen that the Pareto distribution roughly follows a power law at least for $N>10^{5}$, i.e. $f(N) \propto N^{-0.4}$; this is not the case for the WCE data set, Fig. 1(d), where the linearity is present only in a log-linear plot. Notice that the former law exponent of the Pareto distribution is similar to that found in language studies [7]. Such an empirical non trivial power law is consistent with a preferential attachment process [10 on a network, i.e. it is more likely that one has the religion of one's mother or neighbor.... 


\section{Partial distribution functions}

In order to compare the two data sets, and their meaning or bias, and observe the time evolution of adherence (or attachment) we have divided the population interval $\left[1,10^{9}\right]$ into 18 bins of exponentially increasing size and filled each bin with the number of religions having that number of adherents (normalized to have the distribution area equal to 1 ). The result is a partial distribution function (pdf), Fig 2 that can be fitted (i) with a Weibull distribution (symbol + ), much used in life time (or failure) studies,

$$
f(x)=\frac{1}{\beta} e^{-\frac{(x-\mu)}{\beta}} e^{-e^{-\frac{(x-\mu)}{\beta}}}
$$

where $x=\log _{10}(n)$ and $n$ is the number of adherents or/and (ii) with a lognormal distribution (symbol $\mathrm{x}$ ); both fits are quite similar, with a slight difference in the upper tail. For comparison the best corresponding Gaussian distribution (continuous line) is shown in the same plot. This leads to consider that two empirical functions can be possible based on different concepts at this level of data acquisition : (i) birth-death processes? 2 , (ii) multiplicative production with many independent factors.

The same procedure can be applied to the WCE data set, whence obtaining the pdf's shown in Fig. 3 for different (so called) years. To eliminate the effect due to increasing world population in the "years" considered, all pdf's of different "years" were normalized to the same population number considering 1900 as the reference population. A fit of these distributions, with Eq.(11), is shown in Fig 3. In order to plot all the pdf's on the same graph each pdf has been successively displaced by 0.6. The apparent flatness of the pdf is due to the vertical rescaling. From this figure a critical view of this data has to be implied: notice the break at $10^{7}$, indicating in our view an overestimation of adepts/adherents in the most prominent religions, or a lack of distinctions between denominations, as can be easily understood [11. This "emphasis" of the "winner takes all" in the WCE data, i.e. the empirical data results from summing up adherents from the most important sort of religion and smaller related denominations into a single number corresponding to (the main) religion, hints to explaining the difference between Pareto plots in Figs. 1(b), - 1(d),

\section{Time evolution}

Finally, it is easily accepted that the percentages of adherents are not fixed over time. Therefore a nucleation-growth-death process can be proposed, in analogy with crystal growth studies [12. We consider that a microscopic-like, continuous

\footnotetext{
${ }^{2}$ We realize that $x$ is Eq.(1) is the size of the population, while the variable of the Weibull distribution is rather the strength of to-be-broken bonds in a "time to failure" analysis. If there is a one-to-one correspondence between the $x$ and $y$ axes in cause-effect relations, such a change in meaning is only a change in notations. Otherwise, hysteresis effects are to be considered. This goes beyond our present study.
} 
time differential equation can be written for the evolution of the number of adherents (in terms of percentage with respect to the world population) of the world main religions, as for competing entities of the type [13]

$$
\frac{d}{d t} g(t)=S k(t)[1-g(t)] \frac{d V_{n}}{d t}
$$

where, adapting to our case this Avrami-Kolmogorov equation, $g(t)$ is counting the fraction of adherents of a given religion, $V_{n}$ is instead connected with the total world population, $S$ is a parameter to be determined and $k(t) \propto t^{-h}$ where $h$ is a parameter to be deduced in each case, measuring the attachment-growth (or death) process in this continuous time approximation. This should be contrasted with the Lotka-Volterra-Verhulst mechanistic approach (for languages) which hardly allows for nucleation, dissipation and/or time delayed correlations of different entities, in contrast to generalizations of Eq. (2) using such physical features.

A few examples of religions for which the number of adherents is increasing (e.g., Islam), decaying (e.g., Ethnoreligions and Buddhists) or rather stable (e.g., Christianity) is shown in Fig 4 The data can be well fitted to the solution of the Avrami-Kolmogorov growth-death equation Eq.(2). The values of $h$ for the considered religions, as obtained by a least-square best fit, are reported in the plot. The parameter $h$ values and their meaning deserve some short explanation and discussion. The parameter can be thought to be like a reproduction rate in Verhulst logistic equation, or a true attachment like in sexual networks 14 or in molecular processes [15. It is interesting to observe that $h$ can be positive or negative, indicating also the possibility for detachment. Other parametrizations of $k(t)$ can be imagined and are possible. Our theoretical law elsewhere derived from first principles [13] concludes the present scientific analysis in showing that a predictability level can be reached on the evolutions.

\section{Conclusions}

In conclusion, as for languages or wealth, one can recognize religions as a signature of population dynamics. Even though characteristic time scales are different, and religion dynamics is more complex than language dynamics because of the presence of external fields and spontaneous nucleations, empirical ranking laws look similar. Therefore similar growth-death agent based models can be thought of. Yet, there are useful differences to be expected (and found) which lead to different models from those describing language death and appearance. We propose an algorithmic approach based on attachment processes for the macroscopic point of view, - not deciding on the statistical alternative, i.e. Weibull or log-normal law, and a diffusion growth rate based equation for modeling the data at the microscopic level. There are possible open problems on the ongoing research, or further investigations taking into account the available/reliable data at this time, as to look for (time dependent) geographical effects, like clustering, or through other definitions, like normalizing with respect 
to some population size or country surface, or GDP, or other socio-economic index allowing to build correlation matrices and search for socio-economic field influence.

\section{Acknowledgments}

The work by FP has been supported by European Commission Project E2C2 FP6-2003-NEST-Path-012975 Extreme Events: Causes and Consequences. Critical and encouraging comments by A. Morelli have been very valuable. Referees should be thanked, moreover for their warning and putting pressure on us to emphasize that we mere treat religions and adherents as physics variables, so that our results and their interpretation have never the intention of vilifying any religion, sect, person, etc.

\section{References}

[1] R. Axelrod, J Confl. Res. 41 (1997) 203.

[2] D.M. Abrams, S.H.Strogatz, Nature 424 (2003) 900.

[3] E. Bonabeau, G.Theraulaz, J.L.Deneubourg, Physica A 217 (1995) 373.

[4] A.O. Sousa, D. Stauffer, Int. J. Mod. Phys. C 11 (2000) 1063.

[5] C. Tsallis, A.C.N. de Magalhaes, Phys. Reports 268 (1996) 305; A.R.R.Papa, C. Tsallis, Phys. Rev. E 57 (1998) 3923.

[6] J. Holyst, K. Kacperski, F. Schweitzer, Physica A 285 (2000) 199.

[7] V.M. de Oliveira, M.A.F. Gomes, I.R. Tsang, Physica A 361 (2006) 361.

[8] The International Data Base (IDB) is a computerized source of demographic and socioeconomic statistics for 227 countries and areas of the world. The IDB provides a quick access to specialized information, with emphasis on demographic measures, for individual countries or selected groups of countries in the world. The major types of data available in the IDB include: Population by age and sex, Vital rates, infant mortality, and life tables, Fertility and child survivorship, Migration, Marital status, Family planning, Ethnicity, religion, and language, Literacy, Labor force, employment, and income, Households. Sources of the data include: U.S. Census Bureau, Estimates and Projections, National Statistics Offices, United Nations and Specialized Agencies (ILO, UNESCO, WHO))

[9] D. Barrett, G. Kurian, T. Johnson, T. World Christian Encyclopedia (2nd edition). New York: Oxford University Press. (2001) 
[10] R. Albert, A.L. Barabási, A.-L., Phys. Rev. Lett. 85 (2003) 52340.

[11] A. Morelli, private communication.

[12] R. Cloots, N. Vandewalle, M. Ausloos, J. Cryst. Growth 166 (1996) 816.

[13] A. Gadomski, J. Phys. II France 6 (1996) 1537.

[14] J. H. Jones and M. S. Handcock, Proc. R. Soc. Lond. B 270 (2003) 1123.

[15] M. Ausloos, N. Vandewalle and R. Cloots, Phil. Mag. Lett. 73 (1996) 101. 


\section{Figure Captions}

Figure 1 - Zipf's and Pareto's distributions. Subplots (a) and (c) show the Zipf's distribution for the IDB and WCE data sets respectively. On the $y$ axis is the number of adherents; on the $x$ axis the ranked religions. Subplots (b) and (d) show the Pareto distributions for these data sets. These plots show the number of religions ( $y$ axis) with a number of adherents $n>N$ as function of $N$. The axis scales have been chosen to enlighten linear regions

Figure 2 - Partial Distribution Function (pdf) of adherents. The distribution of the number of adherents of religions from the IDB dataset is shown (squares); an exponentially increasing bin size is used for the $x$-axis. The pdf is fitted with Weibull $(+)$ or lognormal $(\mathrm{x})$ distributions and compared with the best Gaussian fit (continuous line).

Figure 3 - Time evolution of Partial Distribution Functions of religion sizes. The distribution of the number of adherents of religions from WCE data set is shown according to an exponentially increasing bin size on the $x$-axis. Results for different "years" are vertically displaced of 0.6 in order to have them on the same plot. The fit is done using a Weibull distribution (continuous lines).

Figure 4 - Time evolution of adherents from the WCE data set. The plot shows the percentage of adherents for 4 typical world religions as a function of time. Each value of the attachment parameter $h$ as given by the best fit is reported in the plots. 
(a)

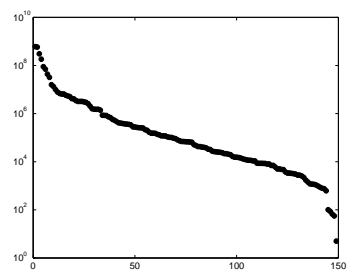

(b)

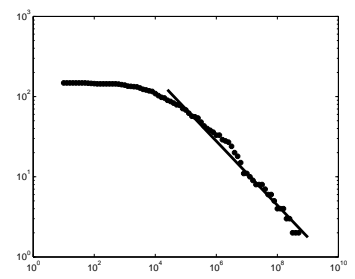

(c)

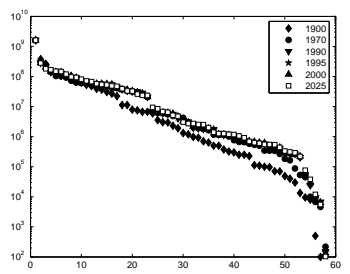

(d)

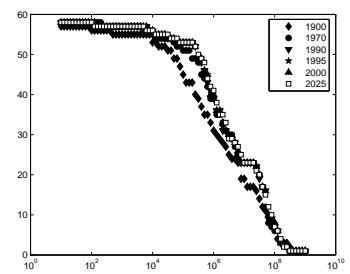

Figure 1: Zipf's and Pareto's distributions of religions. Subplots (a) and (c) show the Zipf's distribution for the IDB and WCE data sets respectively. On the $y$ axis is the number of adherents; on the $x$ axis the ranked religions.Subplots (b) and (d) show the Pareto distributions for these data sets. These plots show the number of religions ( $y$ axis) with a number of adherents $n>N$ as function of $N$. The axis scales have been chosen to enlighten linear regions 


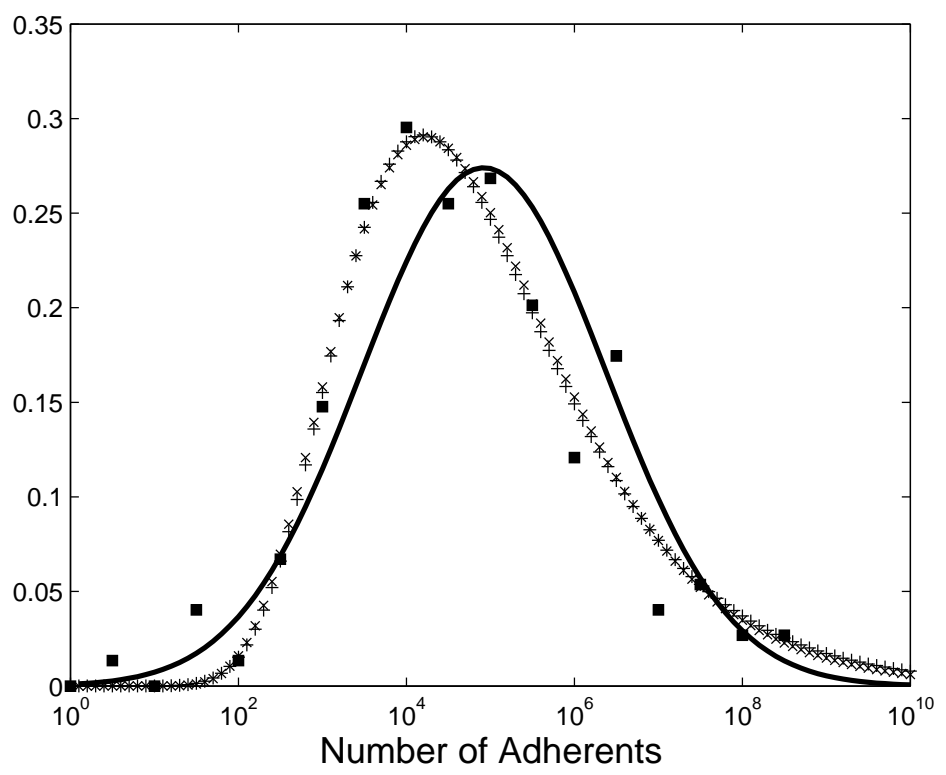

Figure 2: Partial Distribution Function (pdf) of adherents. The distribution of the number of adherents of religions from the IDB dataset is shown (squares); an exponentially increasing bin size is used for the $x$-axis. The pdf is fitted with Weibull $(+)$ or log-normal $(\mathrm{x})$ distributions and compared with the best Gaussian fit (continuous line). 


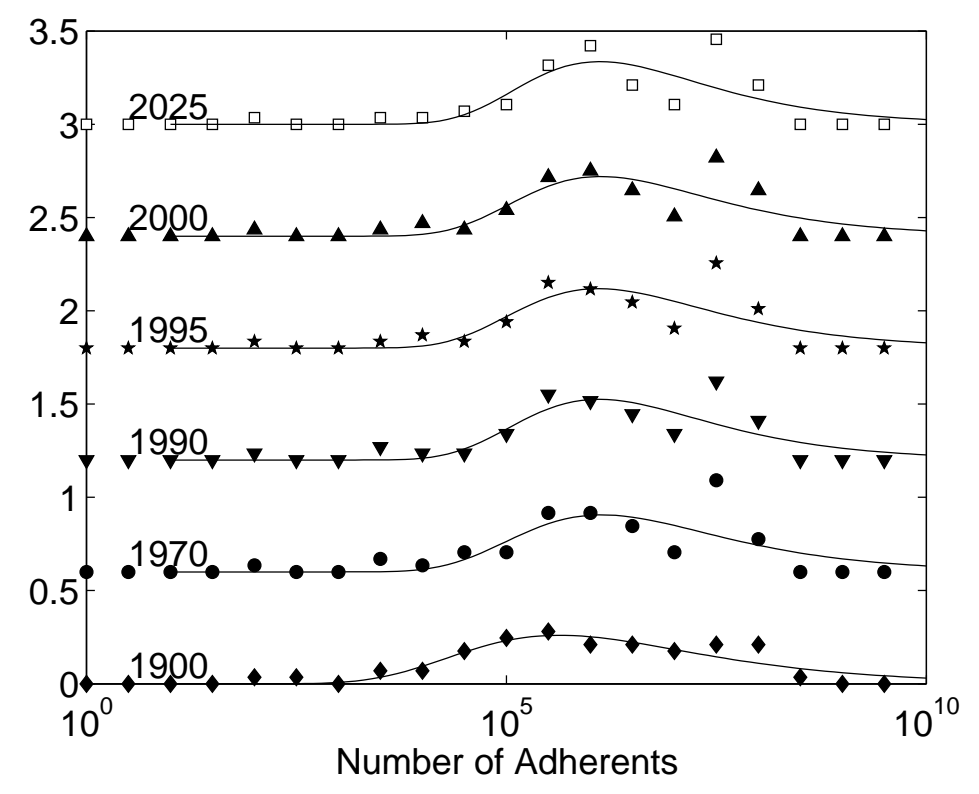

Figure 3: Time evolution of Partial Distribution Functions of religion sizes. The distribution of the number of adherents of religions from WCE data set is shown according to an exponentially increasing bin size on the $x$-axis. Results for different "years" are vertically displaced of 0.6 in order to have them on the same plot. The fit is done using a Weibull distribution (continuous lines). 

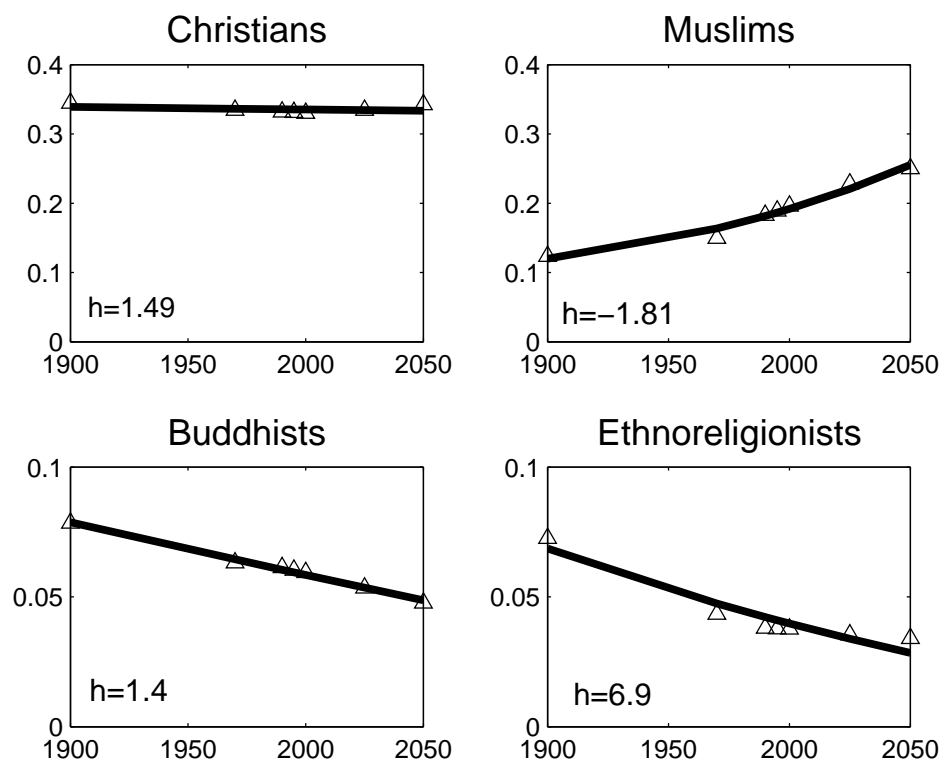

Figure 4: Time evolution of adherents from the WCE data set. The plot shows the percentage of adherents for 4 typical world religions as a function of time. Each value of the attachment parameter $h$ as given by the best fit is reported in the plots 